\title{
MODULI FOR SPECIAL RIEMANN SURFACES OF GENUS 2
}

\author{
BY \\ JOHN SCHILLER
}

Introduction. This paper is an investigation of moduli for the 2-complex parameter family of Riemann surfaces of genus 2 that admit of automorphisms (conformal self-homeomorphisms) of order 2 other than the "interchange of sheets". The determination of these special surfaces is due to Oskar Bolza whose result is contained in §I. In §II characteristic Riemann matrices for the surfaces are imbedded into the Siegel 3-complex dimensional upper half plane, the image being homeomorphic to

$$
\mathscr{J}=H \times H-\{(\tau, \mu \theta \tau) \mid \tau \in H, \mu \in M(2)\},
$$

where $H$ denotes the complex upper half plane, $\theta$ is the bilinear transformation $\tau \rightarrow-1 / \tau$, and $M(2)$ is the even modular group. The transformation group $M_{\mathscr{I}}$ acting on $\mathscr{J}$, whose orbits are all the characteristic matrices for conformally equivalent surfaces, is determined to be a semidirect product of $M_{\mathscr{J}}^{\prime}$ by $Z_{2}$, where

$$
M_{\mathscr{J}}^{\prime}=\{(\mu, \nu) \in M \times M \mid \nu=\theta \mu \theta \bmod M(2)\},
$$

$M$ denoting the classical inhomogeneous group, and $Z_{2}$ is the two-element group. In §III special Teichmueller and Torelli moduli and modular groups are defined for the surfaces. The Teichmueller space is homeomorphic to $H \times H$, and the Torelli space is determined to be homeomorphic to $\mathscr{J}$ of $\S$ II. The Torelli modular group becomes isomorphic to $M_{\mathscr{J}}^{\prime}$ of $\S \mathrm{II}$, and it is shown that the Teichmueller modular group can be constructed as a semidirect product of the fundamental group of $\mathscr{J}$ by $M_{\mathscr{J}}^{\prime}$.

I wish to thank Professor Gerstenhaber who directed the research and whose stimulating lectures on Teichmueller theory have strongly influenced my thinking on the subject.

I. Preliminary notions. Every compact Riemann surface is the Riemann surface of an algebraic function. More precisely, let $S$ be a compact Riemann surface of genus $g$, and let $z$ be a nonconstant meromorphic function on $S$. Then $z$ is an $n$-to-1 surjection from $S$ to the Riemann sphere $P$ for some integer $n \geqq 1$. If we denote by $C$ the complex field as well as the field of constant functions on $S$, and by $C(z)$ the transcendental extension of $C$ consisting of all rational functions of $z$, then the field $K$ of meromorphic functions on $S$ is an algebraic extension of $C(z)$

Received by the editors December 19, 1966 and, in revised form, February 3, 1969. 
of degree $n$, i.e., $K=C(z, w)$, where $w$ is a meromorphic function on $S$ satisfying $f(z, w)=0$, where $f(z, w)$ is a (monic) irreducible polynomial of degree $n$ over $C(z)$. If, by an abuse of notation, we now let $z$ and $w$ denote complex numbers, then $f(z, w)=0$ defines an algebraic function over $C$ whose concrete Riemann surface $\{(z, w)\}$ is conformally equivalent to $S$. Then, if $S$ is identified with $\{(z, w)\}$ (with the resulting duplication of notation), the meromorphic functions $z$ and $w$ on $S$ become the projections $(z, w) \rightarrow z$ and $(z, w) \rightarrow w(z)$, respectively, and $z$ serves to make $S$ an $n$-sheeted branched analytic covering of $P$.

If $g \geqq 1$ and for some $z, n=2$, then $S$ is called hyperelliptic in which case the equation for $S$ becomes, for a suitably chosen $w$,

$$
w^{2}=\left(z-e_{1}\right)\left(z-e_{2}\right) \cdots\left(z-e_{k}\right),
$$

where either $k=2 g+2$ in which case the $e_{i}$ are all the (distinct) images of branch points of $S$ over $P$, or $k=2 g+1$ in which case $\infty$ is also under a branch point. Every Riemann surface of genus 2 is hyperelliptic, and on any hyperelliptic surface $S$ the involutive "interchange of sheets"

$$
\iota:(z, w) \rightarrow(z,-w)
$$

is an automorphism (conformal self-homeomorphism) leaving precisely the $2 g+2$ branch points of $S$ over $P$ fixed. In fact $\iota$ is canonically determined as the only involution on $S$ with $2 g+2$ fixed points, and $(1, \imath)$ is a normal subgroup of Aut $S$, the group of automorphisms on $S$.

By a special surface $S$ of genus 2 we mean one for which Aut $S /(1, \imath)$ is not trivial. Bolza [1] proved that every conformal equivalence class of special surfaces of genus 2 is represented by one of the following cases:

\section{$S$}

$$
\begin{aligned}
& w^{2}=z^{6}-1 \\
& w^{2}=z^{5}-1 \\
& w^{2}=z\left(z^{4}-1\right) \\
& w^{2}=\left(z^{3}-1\right)\left(z^{3}-r^{3}\right) \\
& w^{2}=z\left(z^{2}-1\right)\left(z^{2}-r^{2}\right) \\
& w^{2}=\left(z^{2}-1\right)\left(z^{2}-r_{1}^{2}\right)\left(z^{2}-r_{2}^{2}\right)
\end{aligned}
$$

Aut $S /(1, \imath)$
$D_{6}$
$Z_{5}$
$S_{4}$
$D_{3}$
$D_{2}$
$Z_{2}$

where $D_{n}$ denotes the dihedral group of order $2 n, S_{n}$ the symmetric group on $n$ objects, and $Z_{n}$ the integers $\bmod n$. Observe that the two-complex parameter family of equivalence classes with group $Z_{2}$ contains each of the two one-complex parameter families with groups $D_{2}$ and $D_{3}$, respectively, and these two one-complex parameter families intersect in the classes with groups $S_{4}$ and $D_{6}$, respectively, while the class with group $Z_{5}$ is disjoint from all the others.

$\S$ II and III of this paper are an investigation of the parameters, otherwise called moduli, determining the family with group $Z_{2}$. 
II. Siegel moduli. The holomorphic differentials on a compact Riemann surface of genus $g$ form a $g$-dimensional vector space over the complex field $C$, and if $S$ is a hyperelliptic surface given by

$$
w^{2}=\left(z-r_{1}\right)\left(z-r_{2}\right) \cdots\left(z-r_{2 g+i}\right), \quad i=1 \text { or } 2,
$$

then a basis for the holomorphic differentials on $S$ is $d z / w, z d z / w, \ldots, z^{g-1} d z / w$ where, as in $\S \mathrm{I}, S$ is identified with the concrete surface $\{(z, w)\}$ of $\left(^{*}\right)$, so that on $S$ $z$ and $w$ denote the meromorphic functions $(z, w) \rightarrow z$ and $(z, w) \rightarrow w(z)$, respectively. The $g \times 2 g$ matrix:

$$
(A B) \equiv\left(\begin{array}{cc}
\int_{a_{1}} \frac{d z}{w}, \ldots, \int_{a_{g}} \frac{d z}{w} & \int_{b_{1}} \frac{d z}{w}, \ldots, \int_{b_{g}} \frac{d z}{w} \\
\vdots & \vdots \\
\int_{a_{1}} \frac{z^{g-1} d z}{w}, \ldots, \int_{a_{g}} \frac{z^{g-1} d z}{w} & \int_{b_{1}} \frac{z^{g-1} d z}{w}, \ldots, \int_{b_{g}} \frac{z^{g-1} d z}{w}
\end{array}\right)
$$

where $\left(a_{1}, \ldots, a_{g}, b_{1}, \ldots, b_{g}\right)$ are one-cycle representatives of a canonical homology basis for $S$, is called a period matrix for $S$. By a change in basis for the holomorphic differentials, the matrix $A$ can be reduced to the multiplicative identity, and then $B$ becomes $A^{-1} B$, which is called the Riemann matrix for $S$ with respect to the given canonical homology basis. Every Riemann matrix is symmetric and has positive-definite imaginary part and therefore represents a point in the Siegel $g(g+1) / 2$ complex dimensional upper half plane $\mathscr{S}(g)$, which is precisely the space of all symmetric $g \times g$ matrices over $C$ having positive-definite imaginary part. Not all Siegel matrices, however, are Riemann matrices. In genus 2 it is known [2] that a Siegel matrix $m$ is a Riemann matrix for some surface $S$ if and only if $m$ is not in the orbit of a diagonal matrix under the action of the Siegel inhomogeneous modular group $M_{\mathscr{S}}^{2}$.

A homology basis with one-cycle representatives $\left(a_{1}, \ldots, a_{g}, b_{1}, \ldots, b_{g}\right)$ is called canonical if $I\left(a_{i}, b_{j}\right)=\delta_{i j}$ and $I\left(a_{i}, a_{j}\right)=0=I\left(b_{i}, b_{j}\right)$, where $I$ is the bilinear, skewsymmetric, integral-valued intersection number, and $i, j \in\{1,2, \ldots, g\}$. We refer to a set of one-cycle representatives of a canonical homology basis as a set of retrosections. Then all possible Riemann matrices for a given surface $S$, indeed for all surfaces conformally equivalent to $S$, are obtained by all possible changes in a given set of retrosections for $S$. More precisely, the multiplicative group of matrices of changes in retrosections acts on $\mathscr{S}(g)$, the orbit of any one Riemann matrix for a given conformal equivalence class of Riemann surfaces being all the Riemann matrices for that class. The inhomogeneous Siegel modular group $M_{\mathscr{S}}^{g}$ acting on $\mathscr{S}(g)$ consists of all transformations

$$
Z \rightarrow(A Z+B)(C Z+D)^{-1}, \quad Z \in \mathscr{S}(g),
$$

where $A, B, C, D$ are $g \times g$ matrices over the integers satisfying $A B^{t}=B A^{t}$, $C D^{t}=D C^{t}, A D^{t}-B C^{t}=1$, where $X^{t}$ denotes the transpose of the matrix $X$, and 
1 is the $g \times g$ multiplicative identity matrix. $M_{\mathscr{S}}^{g}$ acts on $\mathscr{S}(g)$ in precisely the same way as the multiplicative group of matrices of changes in retrosections, with the above Siegel transformation corresponding to the change in retrosections

$$
\left(a_{1}, \ldots, a_{g}, b_{1}, \ldots, b_{g}\right) \rightarrow\left(a_{1}^{\prime}, \ldots, a_{g}^{\prime}, b_{1}^{\prime}, \ldots, b_{g}^{\prime}\right),
$$

where

$$
\left(a_{1}^{\prime}, \ldots, a_{g}^{\prime}, b_{1}^{\prime}, \ldots, b_{g}^{\prime}\right)^{t}=\left(\begin{array}{ll}
D & C \\
B & A
\end{array}\right)\left(a_{1}, \ldots, a_{g}, b_{1}, \ldots, b_{g}\right)^{t}
$$

We now restrict our attention to the two-complex parameter family $\{S\}^{\prime}$ of Riemann surfaces of genus 2 that admit of automorphisms of order 2 other than the interchange of sheets. From $\S I$ we see that each $S$ in $\{S\}^{\prime}$ is given by an equation (normalized so that the product of the roots is -1 ) of the form $w^{2}=z^{6}+3 C z^{4}$ $+3 C^{\prime} z^{2}+1=\left(z^{2}-r_{1}^{2}\right)\left(z^{2}-r_{2}^{2}\right)\left(z^{2}-r_{3}^{2}\right)$, and $S$ has at least four automorphisms, namely

$$
\begin{aligned}
1:(z, w) & \rightarrow(z, w), \\
\iota:(z, w) & \rightarrow(z,-w), \quad \text { the interchange of sheets, } \\
\sigma:(z, w) & \rightarrow(-z, w), \\
\iota \sigma:(z, w) & \rightarrow(-z,-w) .
\end{aligned}
$$

Proposition 2.1. $S /(1, \sigma)$ and $S /(1, \iota \sigma)$ are tori.

This follows from the Riemann-Hurwitz relation which says that if a surface $S$ of genus $g$ is an $n$-sheeted branched covering of a surface $S^{\prime}$ of genus $g^{\prime}$, then $2-2 g=n\left(2-2 g^{\prime}\right)-\sum_{P} e_{P}$, where $e_{P}$ denotes the branch order of $P$ in $S$ over $S^{\prime}$. In our case, $g=2$ and $S$ is a 2 -sheeted branched covering of $S /(1, \sigma)$ with exactly two branch points, each of order 1 , located at the fixed points of $\sigma$. Similarly for $\iota \sigma$, so that in both cases $g^{\prime}=1$.

The natural projection $\pi: S \rightarrow S /(1, \iota \sigma)$ is given analytically by

$$
\pi:(z, w) \rightarrow\left(z^{2}, 2 z w\right) \equiv(\zeta, \eta)
$$

so that $S /(1, \iota \sigma)$ has equation

$$
\eta^{2}=4 \zeta\left(\zeta^{3}+3 C \zeta^{2}+3 C^{\prime} \zeta+1\right)=4 \zeta\left(\zeta-r_{1}^{2}\right)\left(\zeta-r_{2}^{2}\right)\left(\zeta-r_{3}^{2}\right),
$$

and the holomorphic differential $d z / w$ on $S$ is a lift of $d \zeta / \eta$ on $S /(1, \iota \sigma)$. Similarly, the natural projection $\hat{\pi}: S \rightarrow S /(1, \sigma)$ is given analytically by $\hat{\pi}:(z, w) \rightarrow\left(z^{2}, 2 w\right)$ $\equiv(\hat{\zeta}, \hat{\eta})$, so that $S /(1, \sigma)$ has equation

$$
\hat{\eta}^{2}=4\left(\hat{\zeta}^{3}+3 C \hat{\zeta}^{2}+3 C^{\prime} \hat{\zeta}+1\right)=4\left(\xi-r_{1}^{2}\right)\left(\hat{\zeta}-r_{2}^{2}\right)\left(\hat{\xi}-r_{3}^{2}\right),
$$

and the holomorphic differential $z d z / w$ on $S$ is a lift of $d \xi / \hat{\eta}$ on $S /(1, \sigma)$.

We can select retrosections $(a, \sigma a, b, \sigma b)$, or equivalently $(a,-\iota \sigma a, b,-\iota \sigma b)$, on $S$, where $(\alpha, \beta) \equiv(\pi a, \pi b)$ are retrosections on $S /(1, \iota \sigma)$, and $(\hat{\alpha}, \hat{\beta}) \equiv(\hat{\pi} a, \hat{\pi} b)$ are 
retrosections on $S /(1, \sigma)$. Now consider a new set of retrosections $\left(\bar{a}_{1}, \bar{a}_{2}, \bar{b}_{1}, \bar{b}_{2}\right)$ on $S$, where

$$
\begin{aligned}
& \bar{a}_{1}=a-\sigma a=a+\imath \sigma a \\
& \bar{a}_{2}=b+\sigma b=b-\imath \sigma b \\
& \bar{b}_{1}=b=b \\
& \bar{b}_{2}=-\sigma a=\imath \sigma a .
\end{aligned}
$$

We hereafter refer to $(a, \sigma a, b, \sigma b)$ and $\left(\bar{a}_{1}, \bar{a}_{2}, \bar{b}_{1}, \bar{b}_{2}\right)$ as normal $\sigma$-retrosections. Observe

$$
\pi:\left(\bar{a}_{1}, \bar{a}_{2}, \bar{b}_{1}, \bar{b}_{2}\right) \rightarrow(2 \alpha, 0, \beta, \alpha)
$$

and

$$
\hat{\pi}:\left(\bar{a}_{1}, \bar{a}_{2}, \bar{b}_{1}, \bar{b}_{2}\right) \rightarrow(0,2 \hat{\beta}, \hat{\beta},-\hat{\alpha})=(0,2 \tilde{\alpha}, \tilde{\alpha}, \tilde{\beta})
$$

where $(\tilde{\alpha}, \tilde{\beta}) \equiv(\hat{\beta},-\hat{\alpha})$ is another set of retrosections on $S /(1, \sigma)$. Hence, the period matrix $(A B)$ for $S$ with respect to $\left(\bar{a}_{1}, \bar{a}_{2}, \bar{b}_{1}, \bar{b}_{2}\right)$ is equal to

$$
\left(\begin{array}{cccc}
2 \int_{\alpha} \frac{d \zeta}{\eta} & 0 & \int_{\beta} \frac{d \zeta}{\eta} & \int_{\alpha} \frac{d \zeta}{\eta} \\
0 & 2 \int_{\tilde{\alpha}} \frac{d \hat{\zeta}}{\hat{\eta}} & \int_{\tilde{\alpha}} \frac{d \hat{\zeta}}{\hat{\eta}} & \int_{\tilde{\beta}} \frac{d \zeta}{\hat{\eta}}
\end{array}\right)=\left(\begin{array}{cccc}
2 \omega & 0 & \omega^{\prime} & \omega \\
0 & 2 \tilde{\omega} & \tilde{\omega} & \tilde{\omega}^{\prime}
\end{array}\right),
$$

and, letting $\tau=\omega^{\prime} / \omega, \tilde{\tau}=\tilde{\omega}^{\prime} / \tilde{\omega}$, the corresponding Riemann matrix $A^{-1} B$ is

$$
\left(\begin{array}{ll}
\frac{1}{2} \tau & \frac{1}{2} \\
\frac{1}{2} & \frac{1}{2} \tau
\end{array}\right)
$$

We denote matrices in $\mathscr{S}(2)$ of this form by $(\tau, \tilde{\tau})$ and call them normal matrices. Hence, every $S$ in $\{S\}^{\prime}$ has a normal matrix representation in $\mathscr{S}(2)$. This result was obtained by Bolza [1] and Hutchinson [3] in a somewhat similar manner( $\left.{ }^{1}\right)$. Note that

$$
(a, \sigma a, b, \sigma b)^{t}=\left(\begin{array}{rrrr}
1 & 0 & 0 & -1 \\
0 & 0 & 0 & -1 \\
0 & 0 & 1 & 0 \\
0 & 1 & -1 & 0
\end{array}\right)\left(\bar{a}_{1}, \bar{a}_{2}, \bar{b}_{1}, \bar{b}_{2}\right)^{t}
$$

so that the Riemann matrix for $S$ with respect to the retrosections $(a, \sigma a, b, \sigma b)$ is

$$
\left(\begin{array}{lr}
(\tau-1 / \tilde{\tau}) / 2 & (-\tau-1 / \tilde{\tau}) / 2 \\
(-\tau-1 / \tilde{\tau}) / 2 & (\tau-1 / \tilde{\tau}) / 2
\end{array}\right)
$$

( ${ }^{1}$ ) The result has since been extended [6] to $g>2$. 
Matrices of this form, i.e., matrices of the form

$$
\left(\begin{array}{ll}
x & y \\
y & x
\end{array}\right)
$$

will be denoted by $\langle x, y\rangle$. Such matrices constitute another normal form, and the Siegel transformation corresponding to the above change in retrosections carries all matrices of the form $(\tau, \tilde{\tau})$ in $\mathscr{S}(2)$ bijectively to those of the form $\langle x, y\rangle$. We wish to determine the subspace of $\mathscr{S}(2)$ that the normal matrices $(\tau, \tilde{\tau})$ determine.

Note that for $g=1$, the Siegel upper half plane $\mathscr{S}(1)$ is the complex upper half plane $H \equiv\left\{\tau \in C \mid \mathscr{I}_{\tau}>0\right\}$, and the Siegel inhomogeneous modular group $M_{\mathscr{S}}^{1}$ is the classical inhomogeneous modular group $M$ consisting of all bilinear transformations

$$
\tau \rightarrow(m \tau+n) /(p \tau+q),
$$

where $m, n, p$ and $q$ are integers such that $m q-n p=1$. We denote by $M(2)$ the even subgroup of $M$ consisting of all transformations in $M$ with matrix

$$
\left(\begin{array}{ll}
m & n \\
p & q
\end{array}\right)=\left(\begin{array}{ll}
1 & 0 \\
0 & 1
\end{array}\right) \quad(\bmod 2)
$$

$M(2)$ is a normal discontinuous free subgroup of $M$ on the two generators

$$
\tau \rightarrow \tau+2 \text { and } \tau \rightarrow \tau /(2 \tau+1),
$$

and $M / M(2)$ is isomorphic to the symmetric group on three objects. Specifically,

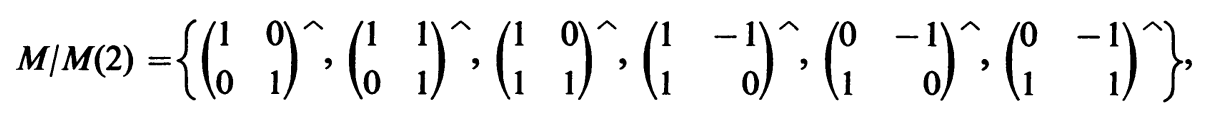

where $(\hat{\mu})$ denotes the equivalence class of the transformation $\mu$ having matrix $(\mu)$.

LEMMA 2.1. Let $S$ in $\{S\}^{\prime}$ have Riemann matrix $(\tau, \tilde{\tau})$ and let $\left(\tau^{\prime}, \tilde{\tau}^{\prime}\right)=(\mu \tau, \nu \tilde{\tau})$, where $(\mu, \nu) \in M(2) \times M(2)$. Then $S$ also has Riemann matrices $\left(\tau^{\prime}, \tilde{\tau}^{\prime}\right),\left(\tilde{\tau}^{\prime}, \tau^{\prime}\right)$.

The Siegel transformation with matrix

$$
\left(\begin{array}{ll}
\left(\begin{array}{ll}
1 & 0 \\
0 & 1
\end{array}\right)\left(\begin{array}{ll}
1 & 0 \\
0 & 0
\end{array}\right) \\
\left(\begin{array}{ll}
0 & 0 \\
0 & 0
\end{array}\right)\left(\begin{array}{ll}
1 & 0 \\
0 & 1
\end{array}\right)
\end{array}\right)
$$

maps $(\tau, \tilde{\tau})$ to $(\tau+2, \tilde{\tau})$, and the transformation with matrix

$$
\left(\begin{array}{ll}
\left(\begin{array}{ll}
1 & 0 \\
2 & 1
\end{array}\right)\left(\begin{array}{rr}
0 & 0 \\
0 & -1
\end{array}\right) \\
\left(\begin{array}{ll}
4 & 0 \\
0 & 0
\end{array}\right)\left(\begin{array}{rr}
1 & -2 \\
0 & 1
\end{array}\right)
\end{array}\right)
$$


maps $(\tau, \tilde{\tau})$ to $(\tau /(2 \tau+1), \tilde{\tau})$, while the transformation with matrix

$$
\left(\begin{array}{ll}
\left(\begin{array}{ll}
0 & 1 \\
1 & 0
\end{array}\right)\left(\begin{array}{ll}
0 & 0 \\
0 & 0
\end{array}\right) \\
\left(\begin{array}{ll}
0 & 0 \\
0 & 0
\end{array}\right)\left(\begin{array}{ll}
0 & 1 \\
1 & 0
\end{array}\right)
\end{array}\right)
$$

$\operatorname{maps}(\tau, \tilde{\tau})$ to $(\tilde{\tau}, \tau)$

LemMA 2.2. Let $(\tau, \tilde{\tau})$ be a normal matrix in $\mathscr{S}(2)$, where $\tilde{\tau}=-1 / \tau \bmod M(2)$. Then no $S$ in $\left\{S^{\prime}\right\}$ has $(\tau, \tilde{\tau})$ for a Riemann matrix.

If some $S$ in $\{S\}^{\prime}$ has $(\tau, \tilde{\tau})$ for a Riemann matrix, where $\tilde{\tau}=-1 / \tau \bmod M(2)$, then, by Lemma 2.1, $S$ also has Riemann matrix $(\tau,-1 / \tau)$, but the Siegel transformation

$$
Z \rightarrow\left(\left(\begin{array}{rr}
1 & 0 \\
-1 & 0
\end{array}\right) Z+\left(\begin{array}{ll}
0 & 0 \\
0 & 1
\end{array}\right)\right)\left(\left(\begin{array}{ll}
0 & -1 \\
0 & -1
\end{array}\right) Z+\left(\begin{array}{ll}
1 & 0 \\
0 & 0
\end{array}\right)\right)^{-1}
$$

maps $(\tau,-1 / \tau)$ onto the diagonal matrix

$$
\left(\begin{array}{ll}
\tau & 0 \\
0 & \tau
\end{array}\right)
$$

and no surface of genus 2 has a diagonal matrix for a Riemann matrix [2].

We now set out to establish the converse of Lemma 2.2. It $T$ is a Riemann surface of genus 1 , then, for a choice of complex numbers $\omega, \omega^{\prime}\left(\omega^{\prime} / \omega=\tau \in H\right)$ determined up to the action of the homogeneous modular group, $T$ is conformally equivalent to the quotient space $C / F\left(\omega, \omega^{\prime}\right)$, where $F\left(\omega, \omega^{\prime}\right)$ is the free abelian group on the two generators $\omega, \omega^{\prime}$. The Weierstrass pe-function $\rho$ and its derivative $\rho^{\prime}$ are meromorphic functions on $\boldsymbol{T}$ satisfying

$$
\begin{aligned}
\left(\wp^{\prime}\right)^{2} & =4 \wp^{3}-g_{2}\left(\omega, \omega^{\prime}\right) \wp-g_{3}\left(\omega, \omega^{\prime}\right) \\
& =\left(4 \wp-e_{1}\right)\left(\wp-e_{2}\right)\left(\wp-e_{3}\right),
\end{aligned}
$$

where $e_{1}=\wp\left(\left(\omega+\omega^{\prime}\right) / 2\right), e_{2}=\varnothing(\omega / 2), e_{3}=\varnothing\left(\omega^{\prime} / 2\right)$ are distinct, so that, in the notation of $\S I, T$ is given by

$$
w^{2}=4 z^{3}-g_{2}\left(\omega, \omega^{\prime}\right) z-g_{3}\left(\omega, \omega^{\prime}\right),
$$

which is called the Weierstrass normal form of the equation of $T$. The even modular function

$$
\lambda(\tau)=\left(e_{1}-e_{2}\right) /\left(e_{1}-e_{3}\right)\left({ }^{2}\right)
$$

(2) The even modular function is usually defined as $1-1 / \lambda$. 
maps $H$ holomorphically onto $C-\{0,1\}$ and is automorphic with respect to $M(2)$, i.e., is well defined, in fact injective, on $H / M(2)$. If $\omega$ and $\omega^{\prime}$ are transformed by the homogeneous modular group to

$$
\hat{\omega}^{\prime}=m \omega^{\prime}+n \omega, \quad \hat{\omega}=p \omega^{\prime}+q \omega,
$$

where $m, n, p$ and $q$ are integers such that $m q-n p=1$, then $\hat{\tau}=\hat{\omega}^{\prime} / \hat{\omega}$ is in $H$, and $g_{i}\left(\hat{\omega}, \hat{\omega}^{\prime}\right)=g_{i}\left(\omega, \omega^{\prime}\right), i=2,3$, but the roots $e_{1}, e_{2}, e_{3}$ are permuted, and conversely. Specifically, there are the following six cases:

(1) $\left(e_{1} e_{2} e_{3}\right) \rightarrow\left(e_{1} e_{2} e_{3}\right)$

$\lambda(\hat{\tau})=\lambda(\tau)$

$\hat{\tau}=\tau$

$\bmod M(2)$

(2) $\left(e_{1} e_{2} e_{3}\right) \rightarrow\left(e_{1} e_{3} e_{2}\right)$,

$\lambda(\hat{\tau})=1 / \lambda(\tau)$,

$\hat{\tau}=-1 / \tau$

$\bmod M(2)$

(3) $\left(e_{1} e_{2} e_{3}\right) \rightarrow\left(e_{2} e_{1} e_{3}\right)$

$\lambda(\hat{\tau})=\lambda(\tau) /(\lambda(\tau)-1)$

$\hat{\tau}=\tau /(\tau+1)$

$\bmod M(2)$

(4) $\left(e_{1} e_{2} e_{3}\right) \rightarrow\left(e_{2} e_{3} e_{1}\right)$,

$\lambda(\hat{\tau})=(\lambda(\tau)-1) / \lambda(\tau), \quad \hat{\tau}=(\tau-1) / \tau$

$\bmod M(2)$

(5) $\left(e_{1} e_{2} e_{3}\right) \rightarrow\left(e_{3} e_{1} e_{2}\right)$,

$\lambda(\hat{\tau})=1 /(1-\lambda(\tau))$,

$\hat{\tau}=-1 /(\tau+1)$

$\bmod M(2)$

(6) $\left(e_{1} e_{2} e_{3}\right) \rightarrow\left(e_{3} e_{2} e_{1}\right)$,

$\lambda(\hat{\tau})=1-\lambda(\tau)$,

$\hat{\tau}=\tau+1$

$\bmod M(2)$.

Finally, we observe that if $T$ has Riemann matrix $(\tau)$ with respect to a given set of retrosections $(\alpha, \beta)$, then

$$
\lambda(\tau)=\left(e_{i_{1}}-e_{i_{2}}\right) /\left(e_{i_{1}}-e_{i_{3}}\right),
$$

where $\left(i_{1} i_{2} i_{3}\right)$ is a permutation on (123) determined by $(\alpha, \beta)$, and if $T^{\prime}$ is another surface of genus 1 with normal form

$$
w^{2}=4\left(z-e_{1}^{\prime}\right)\left(z-e_{2}^{\prime}\right)\left(z-e_{3}^{\prime}\right)
$$

and $\phi: T \rightarrow T^{\prime}$ is a homeomorphism taking $e_{i}$ to $e_{i}^{\prime}$, then

$$
\lambda\left(\tau^{\prime}\right)=\left(e_{i_{1}}^{\prime}-e_{i_{2}}^{\prime}\right) /\left(e_{i_{1}}^{\prime}-e_{i_{3}}^{\prime}\right),
$$

where $\left(\tau^{\prime}\right)$ is the Riemann matrix of $T^{\prime}$ with respect to $(\phi \alpha, \phi \beta)$.

LEMMA 2.3. Let $(a, \sigma a, b, \sigma b)$ be normal $\sigma$-retrosections for an $S$ in $\{S\}^{\prime}$ given by $w^{2}=\left(z^{2}-r_{1}^{2}\right)\left(z^{2}-r_{2}^{2}\right)\left(z^{2}-r_{3}^{2}\right)$. Let $S /(1, \imath \sigma)$ have Riemann matrix $(\tau)$ with respect to the retrosections $(\alpha, \beta) \equiv(\pi a, \pi b)$, where $\pi: S \rightarrow S /(1, \iota \sigma)$ is the natural projection, and let $S /(1, \sigma)$ have Riemann matrix $(\hat{\tau})$ with respect to the retrosections $(\hat{\alpha}, \hat{\beta})$ $\equiv(\hat{\pi} a, \hat{\pi} b)$, where $\hat{\pi}: S \rightarrow S /(1, \sigma)$ is the natural projection. Then

$$
\lambda(\tau)=\frac{r_{i_{3}}^{2} r_{i_{1}}^{2}-r_{i_{2}}^{2}}{r_{i_{2}}^{2} r_{i_{1}}^{2}-r_{i_{3}}^{2}} \text { and } \lambda(\hat{\tau})=\frac{r_{i_{1}}^{2}-r_{i_{2}}^{2}}{r_{i_{1}}^{2}-r_{i_{3}}^{2}}
$$

where $\left(i_{1} i_{2} i_{3}\right)$ is a permutation on (123) determined by $(a, \sigma a, b, \sigma b)$.

$S /(1, \iota \sigma)$ has equation

$$
\eta^{2}=4 \zeta\left(\zeta^{3}+3 C \zeta^{2}+3 C^{\prime} \zeta+1\right)=4 \zeta\left(\zeta-r_{1}^{2}\right)\left(\zeta-r_{2}^{2}\right)\left(\zeta-r_{3}^{2}\right) .
$$

The conformal homeomorphism $\mu:(\zeta, \eta) \rightarrow\left(1 / \zeta+C^{\prime}, \eta / \zeta^{2}\right) \equiv(Z, W)$ maps $S /(1, \iota \sigma)$ onto the Riemann surface $T$ given by the Weierstrass normal form

$$
W^{2}=4 Z^{3}-12\left(C^{\prime 2}-C\right) Z-4\left(3 C C^{\prime}-2 C^{\prime 3}-1\right)=4\left(Z-R_{1}\right)\left(Z-R_{2}\right)\left(Z-R_{3}\right),
$$


where $R_{i}=1 / r_{i}^{2}+C^{\prime}, i=1,2,3$. Then $T$ has Riemann matrix ( $\left.\tau\right)$ with respect to the retrosections $(\mu \alpha, \mu \beta)$, so

$$
\lambda(\tau)=\frac{R_{i_{1}}-R_{i_{2}}}{R_{i_{1}}-R_{i_{3}}}=\frac{r_{i_{3}}^{2}}{r_{i_{2}}^{2}} \frac{r_{i_{1}}^{2}-r_{i_{2}}^{2}}{r_{i_{1}}^{2}-r_{i_{3}}^{2}}
$$

for some permutation $\left(i_{1} i_{2} i_{3}\right)$ on (123). Similarly, $S /(1, \sigma)$ has equation

$$
\hat{\eta}^{2}=4\left(\xi^{3}+3 C \xi^{2}+3 C^{\prime} \xi+1\right)=4\left(\xi+r_{1}^{2}\right)\left(\xi-r_{2}^{2}\right)\left(\xi-r_{3}^{2}\right) \text {, }
$$

and the conformal homeomorphism $v:(\xi, \hat{\eta}) \rightarrow(\xi+C, \hat{\eta}) \equiv\left(Z^{\prime}, W^{\prime}\right)$ maps $S /(1, \sigma)$ onto the Riemann surface $T^{\prime}$ given by the Weierstrass normal form

$$
W^{\prime 2}=4 Z^{\prime 3}-12\left(C^{2}-C^{\prime}\right) Z^{\prime}-4\left(3 C C^{\prime}-2 C^{3}-1\right)=4\left(Z^{\prime}-R_{1}^{\prime}\right)\left(Z^{\prime}-R_{2}^{\prime}\right)\left(Z^{\prime}-R_{3}^{\prime}\right),
$$

where $R_{i}^{\prime}=r_{i}^{2}+C, i=1,2,3$. Then $T^{\prime}$ has Riemann matrix $(\hat{\tau})$ with respect to the retrosections $(\nu \hat{\alpha}, \nu \hat{\beta})$. Furthermore, if $\rho$ denotes the self homeomorphism on $S$ determined by $(a, \sigma a, b, \sigma b) \rightarrow(a,-\sigma a, b,-\sigma b)$, then $\nu \hat{\pi} \rho \pi^{-1} \mu^{-1}: T \rightarrow T^{\prime}$ is a homeomorphism taking $(\mu \alpha, \mu \beta)$ to $(\nu \hat{\alpha}, \nu \hat{\beta})$ and $R_{i}$ to $R_{i}^{\prime}, i=1,2,3$, so that for the same permutation $\left(i_{1} i_{2} i_{3}\right)$ as in the case for $S /(1, \iota \sigma)$,

$$
\lambda(\hat{\tau})=\frac{R_{i_{1}}^{\prime}-R_{i_{2}}^{\prime}}{R_{i_{1}}^{\prime}-R_{i_{3}}^{\prime}}=\frac{r_{i_{1}}^{2}-r_{i_{2}}^{2}}{r_{i_{1}}^{2}-r_{i_{3}}^{2}}
$$

LEMMA 2.4. Let $S$ have Riemann matrix $(\tau, \tilde{\tau})$ with respect to the normal $\sigma$ retrosections $\left(\bar{a}_{1}, \bar{a}_{2}, \bar{b}_{1}, \bar{b}_{2}\right)$. Then

$$
\lambda(\tau)=\frac{r_{i_{3}}^{2}}{r_{i_{2}}^{2}} \frac{r_{i_{1}}^{2}-r_{i_{2}}^{2}}{r_{i_{1}}^{2}-r_{i_{3}}^{2}} \text { and } \lambda(\tilde{\tau})=\frac{r_{i_{1}}^{2}-r_{i_{3}}^{2}}{r_{i_{1}}^{2}-r_{i_{2}}^{2}}
$$

where $\left(i_{1} i_{2} i_{3}\right)$ is a permutation on (123) determined by the $\sigma$-retrosections.

$(\tilde{\tau})$ is the Riemann matrix of $S /(1, \sigma)$ with respect to the retrosections $(\tilde{\alpha}, \tilde{\beta})$ $=(\hat{\beta},-\hat{\alpha})$, and $(\hat{\tau})$ is the Riemann matrix of $S /(1, \sigma)$ with respect to the retrosections $(\hat{\alpha}, \hat{\beta})$. Hence $\tilde{\tau}=-1 / \hat{\tau}$, so $\lambda(\tilde{\tau})=1 / \lambda(\hat{\tau})$. The desired result follows from Lemma 2.3 .

THEOREM 2.1. Let $(\tau, \tilde{\tau})$ be a normal matrix in $\mathscr{S}(2)$. Then there is an $S$ in $\{S\}^{\prime}$ having $(\tau, \tilde{\tau})$ for a Riemann matrix if and only if $\tilde{\tau} \neq-1 / \tau \bmod M(2)$.

Assume $\tilde{\tau} \neq-1 / \tau \bmod M(2)$, and set

$$
\begin{aligned}
& \lambda(\tau)=\frac{r_{3}^{2}}{r_{2}^{2}} \frac{r_{1}^{2}-r_{2}^{2}}{r_{1}^{2}-r_{3}^{2}}=\frac{X_{3}}{X_{2}} \frac{1-X_{2}}{1-X_{3}}, \\
& \lambda(\tilde{\tau})=\frac{r_{1}^{2}-r_{3}^{2}}{r_{1}^{2}-r_{2}^{2}}=\frac{1-X_{3}}{1-X_{2}},
\end{aligned}
$$

where $X_{j}=r_{j}^{2} / r_{1}^{2}, j=2,3$. Now $\lambda: H \rightarrow C-\{0,1\}$, and $\tilde{\tau} \neq-1 / \tau$ means $\lambda(\tilde{\tau}) \neq$ $1 / \lambda(\tau)$, so there is a unique solution $X_{2}, X_{3}$ where $X_{2} \neq 0,1 \neq X_{3} \neq X_{2}$. If $S$ is a corresponding Riemann surface in $\{S\}^{\prime}$, then, for a choice of normal retrosections, 
$S$ has a Riemann matrix $\left(\tau_{1}, \tau_{2}\right)$, where $\lambda\left(\tau_{1}\right)=\lambda(\tau)$ and $\lambda\left(\tau_{2}\right)=\lambda(\tilde{\tau})$, by Lemma 2.4 . By Lemma 2.1 and the fact that $\lambda$ is injective on $H / M(2), S$ also has the Riemann matrix $(\tau, \tilde{\tau})$. The converse is Lemma 2.2.

If we denote by $\mathscr{J}$ the subspace of normal matrices $(\tau, \tilde{\tau})$ of the Siegel upper half plane determined by $\{S\}^{\prime}$, and by $\theta$ the bilinear transformation $\tau \rightarrow-1 / \tau$, then, by Theorem 2.1,

$$
\mathscr{J} \cong H \times H-\{(\tau, \mu \theta \tau) \mid \tau \in H, \mu \in M(2)\} .
$$

We now determine the transformation group $M_{\mathscr{J}}$ acting on $\mathscr{J}$ whose orbits are all the normal matrices for conformally equivalent surfaces in $\{S\}^{\prime} . M_{\mathscr{g}}$ is not a subgroup of the Siegel modular group $M_{\mathscr{S}}^{2}$, but a subquotient, since $\mathscr{J}$ is a space of first category in $\mathscr{S}(2)$ on which $M_{\mathscr{S}}^{2}$ does not act effectively. For example, on the space of normal matrices of the form $\langle x, y\rangle$, the Siegel transformation

$$
Z \rightarrow\left(\begin{array}{ll}
0 & 1 \\
1 & 0
\end{array}\right) Z\left(\begin{array}{ll}
0 & 1 \\
1 & 0
\end{array}\right)
$$

acts as the identity. We now observe the effect of a permutation on $\left(r_{1}^{2} r_{2}^{2} r_{3}^{2}\right)$.

LEMMA 2.5. Let $S$ have Riemann matrix $(\tau, \tilde{\tau})$ with respect to normal $\sigma$-retrosections, and let a permutation on $\left(r_{1}^{2} r_{2}^{2} r_{3}^{2}\right)$ result in the Riemann matrix $\left(\tau^{\prime}, \tilde{\tau}^{\prime}\right)$ for $S$. Then $\tau^{\prime}=\mu \tau$ and $\tilde{\tau}^{\prime}=\nu \tilde{\tau}$, where $(\mu, \nu) \in M \times M$ and $\nu=\theta \mu \theta \bmod M(2)$. Furthermore, all six possibilities for $(\mu, \theta \mu \theta) \bmod M(2)$ occur.

We have, by Lemma 2.4 , that

$$
\lambda(\tau)=\frac{r_{i_{3}}^{2}}{r_{i_{2}}^{2}} \frac{r_{i_{1}}^{2}-r_{i_{2}}^{2}}{r_{i_{1}}^{2}-r_{i_{3}}^{2}} \text { and } \lambda(\tilde{\tau})=\frac{r_{i_{1}}^{2}-r_{i_{3}}^{2}}{r_{i_{1}}^{2}-r_{i_{2}}^{2}}
$$

where $\left(i_{1} i_{2} i_{3}\right)$ is a permutation on (123) determined by the $\sigma$-retrosections. Let $r_{i j}^{2}=X_{j}, j=1,2,3$, and consider the permutation $\left(X_{1} X_{2} X_{3}\right) \rightarrow\left(X_{2} X_{1} X_{3}\right)$. Then

$$
\lambda\left(\tilde{\tau}^{\prime}\right)=\frac{X_{2}-X_{3}}{X_{2}-X_{1}}=1-\frac{X_{1}-X_{3}}{X_{1}-X_{2}}=1-\lambda(\tilde{\tau}),
$$

so $\tilde{\tau}^{\prime}=\tilde{\tau}+1 \bmod M(2)$, and

$$
\lambda\left(\tau^{\prime}\right)=\frac{X_{3}}{X_{1}} \frac{X_{2}-X_{1}}{X_{2}-X_{3}}=\frac{\frac{X_{3}}{X_{2}} \frac{X_{1}-X_{2}}{X_{1}-X_{3}}}{\frac{X_{3}}{X_{2}} \frac{X_{1}-X_{2}}{X_{1}-X_{3}}-1}=\frac{\lambda(\tau)}{\lambda(\tau)-1},
$$

so $\tau^{\prime}=\tau /(\tau+1) \bmod M(2)$. The other cases are similar.

Lemma 2.5 tells the whole story for $\sigma$; i.e., if

$$
\left(\bar{a}_{1}, \bar{a}_{2}, \bar{b}_{1}, \bar{b}_{2}\right)=(a-\sigma a, b+\sigma b, b,-\sigma a)
$$

and

$$
\left(\bar{a}_{1}^{\prime}, \bar{a}_{2}^{\prime}, \bar{b}_{1}^{\prime}, \bar{b}_{2}^{\prime}\right)=\left(a^{\prime}-\sigma a^{\prime}, b^{\prime}+\sigma b^{\prime}, b^{\prime},-\sigma a^{\prime}\right)
$$


are two sets of normal $\sigma$-retrosections on $S$ with corresponding Riemann matrices $(\tau, \tilde{\tau})$ and $\left(\tau^{\prime}, \tilde{\tau}^{\prime}\right)$, respectively, then, by Lemma $2.4,\left(\tau^{\prime}, \tilde{\tau}^{\prime}\right)$ is related to $(\tau, \tilde{\tau})$ as in Lemma 2.5. On the other hand, if we consider normal $\iota \sigma$-retrosections, then an easy computation shows that $(\tau, \tilde{\tau})$ is the Riemann matrix for $S$ with respect to $(a-\sigma a, b+\sigma b, b,-\sigma a)$ if and only if $(\theta \tilde{\tau}, \theta \tau)$ is the Riemann matrix for $S$ with respect to $(a-\imath \sigma a, b+\imath \sigma b, b,-\imath \sigma a)$. We can now establish

THeOREM 2.2. Let $S$ in $\{S\}^{\prime}$ have Riemann matrix $(\tau, \tilde{\tau})$, and let $\left(\tau^{\prime}, \tilde{\tau}^{\prime}\right)$ be a normal matrix in $\mathscr{S}(2)$. Then $\left(\tau^{\prime}, \tilde{\tau}^{\prime}\right)$ is a Riemann matrix for $S$ if and only if either $\left(\tau^{\prime}, \tilde{\tau}^{\prime}\right)=(\mu \tau, \nu \tilde{\tau})$ or $\left(\tau^{\prime}, \tilde{\tau}^{\prime}\right)=(\mu \tilde{\tau}, \nu \tau)$, where $(\mu, \nu) \in M \times M$ and $\nu=\theta \mu \theta \bmod M(2)$.

Suppose $\left(\tau^{\prime}, \tilde{\tau}^{\prime}\right)$ is a Riemann matrix for $S$. As in Theorem 2.1, we can solve the equations for $\lambda\left(\tau^{\prime}\right)$ and $\lambda\left(\tilde{\tau}^{\prime}\right)$ and thereby determine an $S^{\prime}$, where, for a choice of normal retrosections, $S^{\prime}$ has Riemann matrix $\left(\tau_{1}^{\prime}, \tau_{2}^{\prime}\right)$ such that $\left(\tau^{\prime}, \tilde{\tau}^{\prime}\right)=\left(\mu^{\prime} \tau_{1}^{\prime}, \nu^{\prime} \tau_{2}^{\prime}\right)$, where $\left(\mu^{\prime}, \nu^{\prime}\right) \in M(2) \times M(2)$. Then, by Lemma $2.1, S^{\prime}$ also has Riemann matrix $\left(\tau^{\prime}, \tilde{\tau}^{\prime}\right)$, so $S^{\prime}$ is conformally equivalent to $S$, and we may therefore assume that $S^{\prime}$ is $S$. Similarly, we can solve the equations for $\lambda(\tau)$ and $\lambda(\tilde{\tau})$ and thereby determine an $S^{\prime \prime}$, where, for a choice of normal retrosections, $S^{\prime \prime}$ has Riemann matrix $\left(\tau_{1}^{\prime \prime}, \tau_{2}^{\prime \prime}\right)$ such that $(\tau, \tilde{\tau})=\left(\mu^{\prime \prime} \tau_{1}^{\prime \prime}, \nu^{\prime \prime} \tau_{2}^{\prime \prime}\right)$, where $\left(\mu^{\prime \prime}, \nu^{\prime \prime}\right) \in M(2) \times M(2)$, and again, by Lemma 2.1, $S^{\prime \prime}$ also has Riemann matrix $(\tau, \tilde{\tau})$, so $S^{\prime \prime}$ is conformally equivalent to $S$, and we may assume that $S^{\prime \prime}$ is $S$. The normal retrosections involved are either normal $\sigma$-retrosections or normal $\iota \sigma$-retrosections, so either $\left(\tau_{1}^{\prime}, \tau_{2}^{\prime}\right)=\left(\bar{\mu} \tau_{1}^{\prime \prime}, \bar{\nu} \tau_{2}^{\prime \prime}\right)$ or $\left(\tau_{1}^{\prime}, \tau_{2}^{\prime}\right)$ $=\left(\bar{\mu} \tau_{2}^{\prime \prime}, \bar{\nu} \tau_{1}^{\prime \prime}\right)$, where $(\bar{\mu}, \bar{\nu}) \in M \times M$ and $\bar{\nu}=\theta \bar{\mu} \theta \bmod M(2)$. Expressing $\left(\tau^{\prime}, \tilde{\tau}^{\prime}\right)$ in terms of $(\tau, \tilde{\tau})$ via $\left(\tau_{1}^{\prime}, \tau_{2}^{\prime}\right)$ and $\left(\tau_{1}^{\prime \prime}, \tau_{2}^{\prime \prime}\right)$ gives the desired result. Lemmas 2.1 and 2.5 give the converse.

If we denote by $M_{\mathscr{J}}^{\prime}$ the subgroup of $M \times M$ consisting of all $(\mu, \nu)$, where $\nu=\theta \mu \theta \bmod M(2)$, and by $Z_{2}$ the two element group, then Theorem 2.2 tells us that $M_{\mathscr{J}}$ is a semidirect product of $M_{\mathscr{J}}^{\prime}$ by $Z_{2}$, where the action of $M_{\mathscr{J}}^{\prime}$ is $(\tau, \tilde{\tau}) \rightarrow(\mu \tau, \nu \tilde{\tau})$, and the nontrivial action of $Z_{2}$ is $(\tau, \tilde{\tau}) \rightarrow(\tilde{\tau}, \tau)$.

III. Teichmueller and Torelli moduli. The conformal equivalence classes of Riemann surfaces of genus $g \geqq 2$ depend on $3 g-3$ complex parameters (moduli). We present a partial summary of this dependence and then apply the theory to the special surfaces of genus 2 .

Let $f$ and $h$ be homeomorphisms from $S$ onto $S^{\prime}$. We write $f \sim h$ if $f$ is homotopic to $h$. We write $f \approx h$, and say $f$ is homologous to $h$, if $f$ and $h$ induce the same isomorphism from the first homology group of $S$ to that of $S^{\prime}$.

Let $\{S\}$ denote the set of all Riemann surfaces of genus $g \geqq 2$. Define an equivalence relation $R$ on $\{S\}$ by $S R S^{\prime}$ if and only if $S$ is conformally equivalent to $S^{\prime}$. Then $R(g) \equiv\{S\} / R$ is the set of all conformal equivalence classes of surfaces of genus $g$.

Fix $T$ in $\{S\}$ and consider all pairs $\{(S, \phi)\}$, where $S$ is in $\{S\}$ and $\phi: S \rightarrow T$ is an orientation preserving homeomorphism. On $\{(S, \phi)\}$ define two equivalence 
relations $\tilde{R}$ and $\hat{R}$ by $(S, \phi) \tilde{R}\left(S^{\prime}, \phi^{\prime}\right)$ if and only if $\phi^{\prime-1} \phi$ is homotopic to a conformal homeomorphism from $S$ onto $S^{\prime}$, and $(S, \phi) \hat{R}\left(S^{\prime}, \phi^{\prime}\right)$ if and only if $\phi^{\prime-1} \phi$ is homologous to a conformal homeomorphism from $S$ onto $S^{\prime}$. Then $\zeta^{g}(T)$ $\equiv\{(S, \phi)\} / \widetilde{R}$, whose members will be denoted by $(S, \phi)^{\sim}$, is called the Teichmueller space for genus $g$ with respect to the indicatrix $T$, and $\mathscr{T}^{g}(T) \equiv\{(S, \phi)\} \mid \hat{R}$, whose members will be denoted by $(S, \phi)^{\wedge}$, is called the Torelli space for genus $g$ with respect to the indicatrix $T$. Note that $(S, \phi) \tilde{R}\left(S^{\prime}, \phi^{\prime}\right)$ implies $(S, \phi) \hat{R}\left(S^{\prime}, \phi^{\prime}\right)$ which in turn implies $S R S^{\prime}$, so that there are the natural projections

$$
\zeta^{g}(T) \rightarrow \mathscr{T}^{g}(T) \rightarrow R(g) .
$$

Now let $H(T)$ denote the group of orientation preserving homeomorphisms of $T$ onto itself. Then $I \equiv\{h \in H(T) \mid h \sim 1\}$ and $J \equiv\{h \in H(T) \mid h \approx 1\}$ are normal subgroups of $H(T)$. Define $\tilde{H}(T)=H(T) / I$ and denote its members by $\tilde{h}$. Define $\hat{H}(T)=H(T) / J$ and denote its members by $\hat{h}$. For $h$ in $H(T)$ consider the map $(S, \phi) \rightarrow(S, h \phi)$. Then $(S, \phi) \tilde{R}\left(S^{\prime}, \phi^{\prime}\right)$ implies $(S, h \phi) \tilde{R}\left(S^{\prime}, h \phi^{\prime}\right)$, and $f \sim h$ implies $(S, f \phi) \tilde{R}(S, h \phi)$, so $\tilde{H}(T)$ acts on $\zeta^{g}(T)$ by $\tilde{h}:(S, \phi)^{\sim} \rightarrow(S, h \phi)^{\sim}$. Similarly, $\hat{H}(T)$ acts on $\mathscr{T}^{g}(T)$ by $\hat{h}:(S, \phi)^{\wedge} \rightarrow(S, h \phi)^{\wedge}$. Finally, we factor out the trivial action; i.e., let $I^{\prime}=\left\{\tilde{h} \in \tilde{H}(T) \mid(S, h \phi)^{\sim}=(S, \phi)^{\sim}\right.$ for all $\left.(S, \phi)^{\sim}\right\}$, and let

$$
J^{\prime}=\left\{\hat{h} \in \hat{H}(T) \mid(S, h \phi)^{\wedge}=(S, \phi)^{\wedge} \text { for all }(S, \phi)^{\wedge}\right\} .
$$

Then $M_{\zeta}^{g}(T) \equiv \tilde{H}(T) / I^{\prime}$ is called the Teichmueller modular group for genus $g$ with respect to the indicatrix $T$, and $M_{\mathscr{g}}^{g}(T) \equiv \hat{H}(T) / J^{\prime}$ is called the Torelli modular group for genus $g$ with respect to the indicatrix $T$. Then

$$
\zeta^{g}(T) / M_{\zeta}^{g}(T)=R(g)=\mathscr{T}^{g}(T) / M_{\mathscr{S}}^{g}(T) .
$$

Note that $\tilde{h}$ is in $I^{\prime}$ if and only if $(S, h \phi) \tilde{R}(S, \phi)$ for all $(S, \phi)$, i.e., if and only if $\phi^{-1} h \phi$ is homotopic to a conformal homeomorphism on $S$ for all $(S, \phi)$. For $g>2$ not all surfaces admit of automorphisms other than the identity, so for $g>2$ there is an $(S, \phi)$ such that $\phi^{-1} h \phi$ is homotopic to the identity, i.e., $h \sim 1$. Hence $M_{\zeta}^{g}(T)=\tilde{H}(T)$ for $g>2$. Similarly, $M_{\mathscr{F}}^{g}(T)=\hat{H}(T)$ for $g>2$. On the other hand, every surface of genus 2 admits of the conformal interchange of sheets $\iota$. Furthermore, $\iota$ is not homologous (homotopic) to the identity since, for $g \geqq 2$, the only automorphism homologous (homotopic) to the identity is the identity. In fact, $M_{\mathscr{F}}^{g}(T)=\hat{H}(T) /(1, \hat{\imath})$ and $M_{\zeta}^{2}(T)=\tilde{H}(T) /(1, \hat{\imath})$.

Since $h \sim f$ implies $h \approx f$, there is a natural surjective homomorphism $j: \tilde{H}(T)$ $\rightarrow \hat{H}(T)$ defined by $j: \tilde{h} \rightarrow \hat{h}$. Let $G^{g}(T)$ denote the kernel $\{\tilde{h} \in \tilde{H}(T) \mid h \approx 1\}$ of $j$, so that for $g>2$ the sequence

$$
1 \longrightarrow G^{g}(T) \longrightarrow M_{\zeta}^{g}(T) \stackrel{j}{\longrightarrow} M_{\mathscr{F}}^{g}(T) \longrightarrow 1
$$

is exact, and since $j:(1, \tilde{\imath}) \rightarrow(1, \hat{i}), j$ is also defined for genus 2 , again with kernel (isomorphic to) $G^{2}(T)$, so the sequence (3) is also exact for $g=2$, and

$$
\mathscr{T}^{g}(T)=\zeta^{g}(T) / G^{g}(T) .
$$


$\zeta^{g}(T)$ is provided with a metric by means of extremal quasiconformal mappings, and with the resulting topology $\zeta^{g}(T)$ is homeomorphic to $6 g-6$ dimentional euclidean space. $M_{\zeta}^{g}(T)$ is a properly discontinuous group of isometries, and $G^{g}(T)$ is a fixed-point-free subgroup of $M_{\zeta}^{g}(T) . M_{\mathscr{T}}^{g}(T)$ is a properly discontinuous group of homeomorphisms when $\mathscr{T}^{g}(T)$ is given the quotient topology, and then $\zeta^{g}(T)$ is the universal covering of $\mathscr{T}^{g}(T)$ with covering transformation group $G^{g}(T)$, which is then isomorphic to $\pi\left(\mathscr{T}^{g}(T)\right)$, the fundamental group of $\mathscr{T}^{g}(T)$, so

$$
1 \longrightarrow \pi\left(\mathscr{T}^{g}(T)\right) \longrightarrow M_{\zeta}^{g}(T) \stackrel{j}{\longrightarrow} M_{\mathscr{T}}^{g}(T) \longrightarrow 1
$$

is an exact sequence.

Let $\left(a_{1}, \ldots, a_{g}, b_{1}, \ldots, b_{g}\right)$ be a fixed set of retrosections on $T$. Then, given $(S, \phi)$ in $\{(S, \phi)\}, \phi^{-1}\left(a_{1}, \ldots, a_{g}, b_{1}, \ldots, b_{g}\right)$ determines a set of retrosections (elementwise homologous to $\left.\left(\phi^{-1} a_{1}, \ldots, \phi^{-1} a_{g}, \phi^{-1} b_{1}, \ldots, \phi^{-1} b_{g}\right)\right)$ on $S$ and therefore a Riemann matrix $(\gamma)_{\phi}$. If $(S, \phi) \hat{R}\left(S^{\prime}, \phi^{\prime}\right)$, then $(\gamma)_{\phi}=(\gamma)_{\phi^{\prime}}$, so there is a mapping from $\mathscr{T}^{g}(T)$ to the $g(g+1) / 2$ complex dimensional Siegel upper half plane $\mathscr{S}(g)$. In genus 2 this mapping is a bijection from $\mathscr{T}^{2}(T)$ to $\mathscr{S}(2)-D$, where $D$ is the set of all matrices in $\mathscr{S}(2)$ equivalent to diagonal matrices under the action of the Siegel inhomogeneous modular group $M_{\mathscr{S}}^{2}$. Hence,

$$
1 \longrightarrow \pi(\mathscr{S}(2)-D) \longrightarrow M_{\zeta}^{2}(T) \stackrel{j}{\longrightarrow} M_{\mathscr{T}}^{2}(T) \longrightarrow 1
$$

is an exact sequence.

Again, let $\left(a_{1}, \ldots, a_{g}, b_{1}, \ldots, b_{g}\right)$ be a fixed set of retrosections on $T$. Then, if $h$ is in $H(T), h\left(a_{1}, \ldots, a_{g}, b_{1}, \ldots, b_{g}\right)$ determines another set of retrosections $\left(a_{1}^{\prime}, \ldots, a_{g}^{\prime}, b_{1}^{\prime}, \ldots, b_{g}^{\prime}\right)$ on $T$. Let $(\Gamma)_{h}$ be the $2 g \times 2 g$ matrix such that

$$
\left(a_{1}^{\prime}, \ldots, a_{g}^{\prime}, b_{1}^{\prime}, \ldots, b_{g}^{\prime}\right)^{t}=(\Gamma)_{h}\left(a_{1}, \ldots, a_{g}, b_{1}, \ldots, b_{g}\right)^{t},
$$

and let $\Gamma_{h}$ be the corresponding member of the Siegel modular group $M_{\mathscr{S}}^{g}$; i.e.,

$$
(\Gamma)_{h}=\left(\begin{array}{ll}
D & C \\
B & A
\end{array}\right)
$$

and

$$
\Gamma_{h}: Z \rightarrow(A Z+B)(C Z+D)^{-1}, \quad Z \in \mathscr{S}(g) .
$$

If $h \approx f$, then $\Gamma_{h}=\Gamma_{f}$, so $\hat{h} \rightarrow \Gamma_{h}$ is a mapping, in fact a surjective homomorphism, from $\hat{H}(T)$ to $M_{\mathscr{S}}^{g}$. The kernel is $(1, \hat{\imath})$, where $\iota$ is the interchange of sheets, so for genus 2 we have that $M_{\mathscr{T}}^{2}(T)$ is isomorphic to $M_{\mathscr{S}}^{2}$, and hence

$$
1 \longrightarrow \pi(\mathscr{S}(2)-D) \longrightarrow M_{\zeta}^{2}(T) \stackrel{j}{\longrightarrow} M_{\mathscr{S}}^{2} \longrightarrow 1
$$

is an exact sequence. This is, in part, the global picture. For a complete development, including the analytic structure of $\zeta^{g}(T)$, see [5] and the references cited there. 
We now apply the above theory to the special surfaces of genus 2 . Let $\{S\}^{\prime}$ denote (as in §II) all the Riemann surfaces of genus 2 that admit of automorphisms of order 2 other than the interchange of sheets. Select $T$ in $\{S\}^{\prime}$ and let $\sigma_{T}$ be a fixed involution on $T$ that is not homologous to the interchange of sheets. Define the equivalence relation $R$ on $\{S\}^{\prime}$ as in the general case, so that $R^{\prime}(2) \equiv\{S\}^{\prime} / R$ is the set of conformal equivalence classes of the special surfaces of genus 2 . Now consider all pairs $\{(S, \phi)\}^{\prime}$, where $S$ is in $\{S\}^{\prime}$ and $\phi: S \rightarrow T$ is an orientation preserving homeomorphism such that $\phi^{-1} \sigma_{T} \phi$ is homotopic to an automorphism on $S$ (of order 2). Define the equivalence relations $\tilde{R}$ and $\hat{R}$ on $\{(S, \phi)\}^{\prime}$ as in the general case. Then we call $\zeta\left(T, \sigma_{T}\right) \equiv\{(S, \phi)\}^{\prime} \mid \widetilde{R}$ the special Teichmueller space for genus 2 , and $\mathscr{T}\left(T, \sigma_{T}\right) \equiv\{(S, \phi)\}^{\prime} \mid \hat{R}$ the special Torelli space for genus 2 . As in the general case, we have the natural projections

$$
\zeta\left(T, \sigma_{T}\right) \rightarrow \mathscr{T}\left(T, \sigma_{T}\right) \rightarrow R^{\prime}(2) .
$$

Let $H\left(T, \sigma_{T}\right)$ denote all orientation preserving homeomorphisms on $T$ that commute with $\sigma_{T}$ up to homotopy. Then if $h \in H\left(T, \sigma_{T}\right)$ and $(S, \phi) \in\{(S, \phi)\}^{\prime}$, we have $(h \phi)^{-1} \sigma_{T}(h \phi)=\phi^{-1}\left(h^{-1} \sigma_{T} h\right) \phi \sim \phi^{-1} \sigma_{T} \phi$, so $(S, h \phi) \in\{(S, \phi)\}^{\prime}$, and if $I\left(\sigma_{T}\right)$ denotes the normal subgroup of $H\left(T, \sigma_{T}\right)$ consisting of those homeomorphisms homotopic to the identity, then $\tilde{H}\left(T, \sigma_{T}\right) \equiv H\left(T, \sigma_{T}\right) / I\left(\sigma_{T}\right)$ acts on $\zeta\left(T, \sigma_{T}\right)$ by $\tilde{h}:(S, \phi)^{\sim}$ $\rightarrow(S, h \phi)^{\sim}$ as in the general case. Similarly, if $J\left(\sigma_{T}\right)$ denotes the normal subgroup of $H\left(T, \sigma_{T}\right)$ consisting of those homeomorphisms homologous to the identity, then $\hat{H}\left(T, \sigma_{T}\right) \equiv H\left(T, \sigma_{T}\right) / J\left(\sigma_{T}\right)$ acts on $\mathscr{T}\left(T, \sigma_{T}\right)$ by $\hat{h}:(S, \phi)^{\wedge} \rightarrow(S, h \phi)^{\wedge}$. As in the general case, we factor out the trivial action; i.e., let $I^{\prime}\left(\sigma_{T}\right)$ and $J^{\prime}\left(\sigma_{T}\right)$ denote the normal subgroups of $\tilde{H}\left(T, \sigma_{T}\right)$ and $\hat{H}\left(T, \sigma_{T}\right)$, respectively, that leave fixed every point of $\zeta\left(T, \sigma_{T}\right)$ and $\mathscr{T}\left(T, \sigma_{T}\right)$ respectively. It is easily verified that $I^{\prime}\left(\sigma_{T}\right)$ $=\left\{1, \tilde{\imath}, \tilde{\sigma}_{T}, \iota \sigma_{T} \sim\right\}$ and $J^{\prime}\left(\sigma_{T}\right)=\left\{1, \hat{\imath}, \hat{\sigma}_{T}, \iota \sigma_{T} \hat{}\right\}$, where $\iota$ denotes the interchange of sheets on $T$. We call $M_{\zeta}\left(T, \sigma_{T}\right) \equiv \tilde{H}\left(T, \sigma_{T}\right) / I^{\prime}\left(\sigma_{T}\right)$ the special Teichmueller modular group for genus 2 , and $M_{\mathscr{T}}\left(T, \sigma_{T}\right) \equiv \hat{H}\left(T, \sigma_{T}\right) / J^{\prime}\left(\sigma_{T}\right)$ the special Torelli modular group for genus 2 . Clearly,

$$
\zeta\left(T, \sigma_{T}\right) / M_{\zeta}\left(T, \sigma_{T}\right)=\mathscr{T}\left(T, \sigma_{T}\right) / M_{\mathscr{T}}\left(T, \sigma_{T}\right),
$$

whereas we will see later that the surjection

$$
\mathscr{T}\left(T, \sigma_{T}\right) / M_{\mathscr{T}}\left(T, \sigma_{T}\right) \rightarrow R^{\prime}(2)
$$

\section{is 2-to-1.}

As in the general case, the surjective homomorphism $j: \tilde{H}\left(T, \sigma_{T}\right) \rightarrow \hat{H}\left(T, \sigma_{T}\right)$ defined by $j: \tilde{h} \rightarrow \hat{h}$ with kernel $G\left(T, \sigma_{T}\right)=\left\{\tilde{h} \in \tilde{H}\left(T, \sigma_{T}\right) \mid h \approx 1\right\}$ maps $I^{\prime}\left(\sigma_{T}\right)$ onto $J^{\prime}\left(\sigma_{T}\right)$, so $j$ is defined from $M_{\zeta}\left(T, \sigma_{T}\right)$ onto $M_{\mathscr{T}}\left(T, \sigma_{T}\right)$ with kernel equal to $G\left(T, \sigma_{T}\right) I^{\prime}\left(\sigma_{T}\right) / I^{\prime}\left(\sigma_{T}\right)$ which, since $G\left(T, \sigma_{T}\right) \cap I^{\prime}\left(\sigma_{T}\right)=\{1\}$, is isomorphic to $G\left(T, \sigma_{T}\right)$, so

$$
1 \longrightarrow G\left(T, \sigma_{T}\right) \longrightarrow M_{\zeta}\left(T, \sigma_{T}\right) \stackrel{j}{\longrightarrow} M_{\mathscr{T}}\left(T, \sigma_{T}\right) \longrightarrow 1
$$


is an exact sequence. Moreover,

$$
\zeta\left(T, \sigma_{T}\right) / G\left(T, \sigma_{T}\right)=\mathscr{T}\left(T, \sigma_{T}\right) .
$$

With the metric and corresponding topology defined as in the general case, $\zeta\left(T, \sigma_{T}\right)$ is homeomorphic to 4-dimensional euclidean space, $M_{\zeta}\left(T, \sigma_{T}\right)$ is a properly discontinuous group of isometries, and $G\left(T, \sigma_{T}\right)$ is a fixed-point-free subgroup. $M_{\mathscr{T}}\left(T, \sigma_{T}\right)$ is a properly discontinuous group of homeomorphisms when $\mathscr{T}\left(T, \sigma_{T}\right)$ is given the quotient topology, and then $\zeta\left(T, \sigma_{T}\right)$ is the universal covering of $\mathscr{T}\left(T, \sigma_{T}\right)$ with covering transformation group $G\left(T, \sigma_{T}\right)$, which is then isomorphic to $\pi\left(\mathscr{T}\left(T, \sigma_{T}\right)\right)$, the fundamental group of $\mathscr{T}\left(T, \sigma_{T}\right)$, so

$$
1 \longrightarrow \pi\left(\mathscr{T}\left(T, \sigma_{T}\right)\right) \longrightarrow M_{\zeta}\left(T, \sigma_{T}\right) \stackrel{j}{\longrightarrow} M_{\mathscr{T}}\left(T, \sigma_{T}\right) \longrightarrow 1
$$

is an exact sequence.

Now take retrosections $\left(a, \sigma_{T} a, b, \sigma_{T} b\right)$ for a fixed set of retrosections on $T$. Then, given $(S, \phi)$ in $\{(S, \phi)\}^{\prime}, \phi^{-1} a, \phi^{-1}\left(\sigma_{T} a\right), \phi^{-1} b, \phi^{-1}\left(\sigma_{T} b\right)$ are elementwise homologous to a set of retrosections on $S$, and hence a Riemann matrix $(\gamma)_{\phi}$ is determined for $S$. As in the general case for genus $2,(S, \phi) \hat{R}\left(S^{\prime}, \phi^{\prime}\right)$ if and only if $(\gamma)_{\phi}=(\gamma)_{\phi^{\prime}}$, so there is an injective mapping $\mathscr{T}\left(T, \sigma_{T}\right) \rightarrow \mathscr{S}(2)$ defined by $\left(S, \phi^{\wedge}\right) \rightarrow(\gamma)_{\phi}$. Note that, by definition, $\phi^{-1} \sigma_{T} \phi \sim \sigma_{S}$, where $\sigma_{S}$ is an automorphism of order 2 on $S$, so that $\phi^{-1}\left(\sigma_{T} a\right) \approx \sigma_{S}\left(\phi^{-1} a\right)$ and $\phi^{-1}\left(\sigma_{T} b\right) \approx \sigma_{S}\left(\phi^{-1} b\right)$. Hence the retrosections determined by $\phi$ are of the form $\left(a^{\prime}, \sigma_{S} a^{\prime}, b^{\prime}, \sigma_{S} b^{\prime}\right)$, i.e., are normal retrosections. We saw in §II that such normal retrosections give rise to normal Riemann matrices of the form $\langle x, y\rangle$. Furthermore, the Siegel transformation

$$
\Gamma: Z \rightarrow\left(\left(\begin{array}{ll}
1 & 0 \\
0 & 0
\end{array}\right) Z+\left(\begin{array}{rr}
0 & 0 \\
0 & -1
\end{array}\right)\right)\left(\left(\begin{array}{rr}
0 & 0 \\
1 & 1
\end{array}\right) Z+\left(\begin{array}{rr}
1 & -1 \\
0 & 0
\end{array}\right)\right)^{-1}
$$

takes all matrices in $\mathscr{S}(2)$ of the form $\langle x, y\rangle$ bijectively to those of the form $(\tau, \tilde{\tau})$. Hence we have an injective mapping

$$
(S, \phi)^{\wedge} \rightarrow\langle x, y\rangle_{\phi} \rightarrow \Gamma\langle x, y\rangle_{\phi}
$$

from $\mathscr{T}\left(T, \sigma_{T}\right)$ into the space $\mathscr{J}$ of normal matrices of §II. We will soon see (Theorem 3.2) that this mapping is a bijection, but for the moment we denote the image by $\mathscr{J}^{\prime}$, so that

$$
1 \longrightarrow \pi\left(\mathscr{J}^{\prime}\right) \longrightarrow M_{\zeta}\left(T, \sigma_{T}\right) \stackrel{j}{\longrightarrow} M_{\mathcal{F}}\left(T, \sigma_{T}\right) \longrightarrow 1
$$

is an exact sequence.

We now investigate the action of $M_{\mathscr{T}}\left(T, \sigma_{T}\right)$ on $\mathscr{J}^{\prime} . M_{\mathscr{T}}\left(T, \sigma_{T}\right)$ acts on $\mathscr{T}\left(T, \sigma_{T}\right)$ by $\hat{h}:(S, \phi)^{\wedge} \rightarrow(S, h \phi)^{\wedge}$, where $\hat{h}$ is in $\hat{H}\left(T, \sigma_{T}\right)$, so on $\mathscr{J}^{\prime}$ the action is $\hat{h}: \Gamma\langle x, y\rangle_{\phi}$ $\rightarrow \Gamma\langle x, y\rangle_{n \phi}$. If $\left(a^{\prime}, \sigma_{S} a^{\prime}, b^{\prime}, \sigma_{S} b^{\prime}\right)$ (elementwise homologous to $\left(\phi^{-1} a, \phi^{-1} \sigma_{T} a\right.$, $\left.\left.\phi^{-1} b, \phi^{-1} \sigma_{T} b\right)\right)$ are the retrosections giving rise to $\langle x, y\rangle_{\phi}$, then retrosections elementwise homologous to $\left((h \phi)^{-1} a,(h \phi)^{-1} \sigma_{T} a,(h \phi)^{-1} b,(h \phi)^{-1} \sigma_{T} b\right)$ give rise to 
$\langle x, y\rangle_{h \Phi}$. Note that $h \sigma_{T} \sim \sigma_{T} h$ by the definition of $H\left(T, \sigma_{T}\right)$, so that the retrosections giving rise to $\langle x, y\rangle_{h \phi}$ are of the form $\left(a^{\prime \prime}, \sigma_{S} a^{\prime \prime}, b^{\prime \prime}, \sigma_{S} b^{\prime \prime}\right)$, and hence

$$
\left(a^{\prime \prime}, \sigma_{S} a^{\prime \prime}, b^{\prime \prime}, \sigma_{S} b^{\prime \prime}\right)^{t}=(\Gamma)_{h}\left(a^{\prime}, \sigma_{S} a^{\prime}, b^{\prime}, \sigma_{S} b^{\prime}\right)^{t},
$$

where the matrix $(\Gamma)_{h}$ is of the form:

$$
\left(\begin{array}{llll}
m & n & p & q \\
n & m & q & p \\
r & s & u & v \\
s & r & v & u
\end{array}\right) .
$$

In particular,

$$
(\Gamma)_{\iota}=\left(\begin{array}{rrrr}
-1 & 0 & 0 & 0 \\
0 & -1 & 0 & 0 \\
0 & 0 & -1 & 0 \\
0 & 0 & 0 & -1
\end{array}\right) \quad \text { and }(\Gamma) \sigma_{T}=\left(\begin{array}{llll}
0 & 1 & 0 & 0 \\
1 & 0 & 0 & 0 \\
0 & 0 & 0 & 1 \\
0 & 0 & 1 & 0
\end{array}\right)
$$

Let $\Gamma_{h}$ denote the transformation corresponding to $(\Gamma)_{h}$; i.e.,

$$
\Gamma_{h}: Z \rightarrow\left(\left(\begin{array}{ll}
u & v \\
v & u
\end{array}\right) Z+\left(\begin{array}{ll}
r & s \\
s & r
\end{array}\right)\right)\left(\left(\begin{array}{ll}
p & q \\
q & p
\end{array}\right) Z+\left(\begin{array}{ll}
m & n \\
n & m
\end{array}\right)\right)^{-1}
$$

Then $\langle x, y\rangle_{h \phi}=\Gamma_{h}\langle x, y\rangle_{\phi}$ or, denoting $\Gamma\langle x, y\rangle$ by $(\tau, \tilde{\tau})$, the action on $\mathscr{J}^{\prime}$ is $\hat{h}:(\tau, \tilde{\tau}) \rightarrow \Gamma \Gamma_{h} \Gamma^{-1}(\tau, \tilde{\tau})$, and $\Gamma \Gamma_{h} \Gamma^{-1}(\tau, \tilde{\tau})$ is equal to

$$
\left(\frac{(u-v) \tau+(r-s)}{(p-q) \tau+(m-n)}, \frac{(m+n) \tilde{\tau}-(p+q)}{-(r+s) \tilde{\tau}+(u+v)}\right)=\left(\mu_{h} \tau, \nu_{h} \tilde{\tau}\right)
$$

in the notation of §II. It is easily verified that $\left(\mu_{h}, \nu_{h}\right) \in M \times M$ and $\nu_{h}=\theta \mu_{h} \theta$ mod $M(2)$, where $\theta$ denotes the bilinear transformation $\tau \rightarrow-1 / \tau$. Hence we have a mapping, in fact a homomorphism, from $H\left(T, \sigma_{T}\right)$ into the group

$$
M_{\mathscr{J}}^{\prime}=\{(\mu, \nu) \in M \times M \mid \nu=\theta \mu \theta \bmod M(2)\}
$$

of §II. On the other hand, given $(\mu, \nu)$ in $M_{\mathscr{J}}^{\prime}$, we can solve uniquely for $m, n, p, q, r$, $s, u, v$ and thus determine an automorphism $(\Gamma)$ of the fundamental group of $T$. Since every automorphism of the fundamental groüp of a compact surface is induced by a homeomorphism of the surface onto itself [5], there is a homeomorphism $h$ of $T$ which induces $(\Gamma)$, and, since $(\Gamma)(\Gamma) \sigma_{T}=(\Gamma) \sigma_{T}(\Gamma)$, it follows that $h \sigma_{T} \sim \sigma_{T} h$, i.e., that $h$ is in $H\left(T, \sigma_{T}\right)$. Hence the homomorphism is onto. The kernel if $J^{\prime}\left(\sigma_{T}\right)$, so we now have

THEOREM 3.1. The special Torelli group $M_{\mathscr{T}}\left(T, \sigma_{T}\right)$ acting on the special Torelli space $\mathscr{T}\left(T, \sigma_{T}\right)=\mathscr{J}^{\prime}$ is isomorphic to $M_{\mathscr{J}}^{\prime}$. For $(\mu, \nu)$ in $M_{\mathscr{J}}^{\prime}$ and $(\tau, \tilde{\tau})$ in $\mathscr{J}^{\prime}$, the action is $(\tau, \tilde{\tau}) \rightarrow(\mu \tau, \nu \tilde{\tau})$. 
Now consider for $T$ the matrix $(\tau, \tilde{\tau})$ in $\mathscr{J}$ arising from the normal retrosections $\left(\bar{a}_{1}, \bar{a}_{2}, \bar{b}_{1}, \bar{b}_{2}\right)$, where

$$
\left(\bar{a}_{1}, \bar{a}_{2}, \bar{b}_{1}, \bar{b}_{2}\right)^{t}=\left(\begin{array}{rrrr}
1 & -1 & 0 & 0 \\
0 & 0 & 1 & 1 \\
0 & 0 & 1 & 0 \\
0 & -1 & 0 & 0
\end{array}\right)\left(a, \sigma_{T} a, b, \sigma_{T} b\right)^{t}
$$

The change in retrosections

$$
\left(\bar{a}_{1}, \bar{a}_{2}, \bar{b}_{1}, \bar{b}_{2}\right) \rightarrow\left(\bar{a}_{2}, \bar{a}_{1}, \bar{b}_{2}, \bar{b}_{1}\right)
$$

takes $(\tau, \tilde{\tau})$ to $(\tilde{\tau}, \tau)$. This change, however, corresponds to

$$
\left(a, \sigma_{T} a, b, \sigma_{T} b\right) \rightarrow\left(\sigma_{T} b,-b,-\sigma_{T} a, a\right),
$$

which, since $\sigma_{T}\left(\sigma_{T} b\right) \neq-b$, does not arise from any $h$ in $H\left(T, \sigma_{T}\right)$, and this explains why the group $Z_{2}$ of §II does not appear, and also why the surjection (2)" is 2-to-1, for we now prove

TheOrem 3.2. $\mathscr{J}^{\prime}=\mathscr{J}$; i.e., the injection from the special Torelli space $\mathscr{T}\left(T, \sigma_{T}\right)$ into the space $\mathscr{J}$ of normal matrices of the form $(\tau, \tilde{\tau})$ is a surjection.

Every $S$ in $\{S\}^{\prime}$ has at least one representative $(\tau, \tilde{\tau})$ in $\mathscr{J}^{\prime}$. Then, by Theorems 2.2 and 3.1 , it suffices to show that $(\theta \tilde{\tau}, \theta \tau)$ is in $\mathscr{J}^{\prime}$ whenever $(\tau, \tilde{\tau})$ is. If $(\tau, \tilde{\tau})$ is in $\mathscr{J}^{\prime}$, then $(\tau, \tilde{\tau})=\Gamma\langle x, y\rangle_{\phi}$, where $\langle x, y\rangle_{\phi}$ is the normal Riemann matrix with respect to the normal retrosections

$$
\left(a^{\prime}, \sigma_{S} a^{\prime}, b^{\prime}, \sigma_{S} b\right) \approx\left(\phi^{-1} a, \phi^{-1} \sigma_{T} a, \phi^{-1} b, \phi^{-1} \sigma_{T} b\right)
$$

for some $(S, \phi)$ in $\{(S, \phi)\}^{\prime}$. Let $\rho$ be the self-homeomorphism on $S$ determined by

$$
\rho:\left(a^{\prime}, \sigma_{S} a^{\prime}, b^{\prime}, \sigma_{S} b^{\prime}\right) \rightarrow\left(a^{\prime},-\sigma_{S} a^{\prime}, b^{\prime},-\sigma_{S} b^{\prime}\right) .
$$

Then $(\phi \rho)^{-1} \sigma_{T}(\phi \rho) \sim \rho^{-1} \sigma_{S} \rho \sim \iota \sigma_{S}$, where $\iota$ is the interchange of sheets on $S$, so $(S, \phi \rho)$ is in $\{(S, \phi)\}^{\prime}$. Furthermore,

$$
\left((\phi \rho)^{-1} a,(\phi \rho)^{-1} \sigma_{T} a,(\phi \rho)^{-1} b,(\phi \rho)^{-1} \sigma_{T} b\right) \approx\left(a^{\prime}, \iota \sigma_{S} a^{\prime}, b^{\prime}, \iota \sigma_{S} b^{\prime}\right),
$$

and if $\langle x, y\rangle_{\phi \rho}$ is the Riemann matrix with respect to these retrosections, it is easily checked (see $\S \mathrm{II})$ that $\Gamma\langle x, y\rangle_{\phi \rho}=(\theta \tilde{\tau}, \theta \tau)$, i.e., that $(\theta \tilde{\tau}, \theta \tau)$ is the Riemann matrix with respect to $\left(a^{\prime}-\iota \sigma_{S} a^{\prime}, b^{\prime}+\iota \sigma_{S} b^{\prime},-\iota \sigma_{S} a^{\prime}\right)$.

Hence,

$$
1 \longrightarrow \pi(\mathscr{J}) \longrightarrow M_{\zeta}\left(T, \sigma_{T}\right) \stackrel{j}{\longrightarrow} M_{\mathscr{J}}^{\prime} \longrightarrow 1
$$

is an exact sequence.

We are now in a position to construct the special Teichmueller modular group $M_{\zeta}\left(T, \sigma_{T}\right)$, but first we recall some basic notions. If $K$ is a group, we denote the 
group of automorphisms of $K$ by Aut $K$, the group of inner automorphisms by Inn $K$, and Aut $K / \operatorname{Inn} K$ by Out $K$. Let $G$ be a group extension of $K$ by $Q$; i.e., for some homomorphism $j$, the sequence

$$
1 \longrightarrow K \longrightarrow G \stackrel{j}{\longrightarrow} Q \longrightarrow 1
$$

is exact, and let $s: \dot{Q} \rightarrow G$ be a mapping of sets such that $j s=1$. Then, given $q$ in $Q$, the mapping

$$
q(s): K \rightarrow K \text { by } q(s): k \rightarrow(s q)^{-1} k(s q)
$$

is in Aut $K$, and if $s^{\prime}$ is another such set mapping, then $q\left(s^{\prime}\right)^{-1} q(s)$ is in Inn $K$, so that if $\bar{q}$ denotes the equivalence class of $q(s)$ in Out $K$, the mapping

$$
\psi: Q \rightarrow \text { Out } K \text { by } \psi: q \rightarrow \bar{q}
$$

is a homomorphism, called the natural homomorphism. On the other hand, given groups $K, Q$ and a homomorphism $\phi: Q \rightarrow$ Out $K$, then an extension $G$ of $K$ by $Q$ is said to realize $\phi$ if $\psi=\phi$, where $\psi$ is the natural homomorphism. In general, there is an obstruction to $\phi$ in the third cohomology group of $Q$ with coefficients in the center of $K$, and even given existence, there are as many extensions realizing $\phi$ as there are elements in the second cohomology group of $Q$ with coefficients in the center of $K$. Of course, if $K$ is a free group, then there is a unique extension realizing $\phi$.

Now, given $K, Q$ and $\phi: Q \rightarrow$ Out $K$ where $K$ is a free group, suppose that $\phi$ can be lifted to $\phi: Q \rightarrow$ Aut $K$, i.e., that $\phi$ is a homomorphism, and

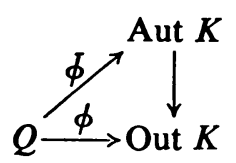

commutes. Then the unique extension $G$ of $K$ realising $\phi$ can be constructed as a semidirect product $K \times{ }_{\phi} Q$, where

$$
K \times{ }_{\circ} Q=(K \times Q, \circ) \text { and }(k, q) \circ\left(k^{\prime}, q^{\prime}\right)=\left(k \phi(q) k^{\prime}, q q^{\prime}\right) .
$$

THEOREM 3.3. The Teichmueller modular group $M_{\zeta}\left(T, \sigma_{T}\right)$ can be constructed as a semidirect product of the fundamental group $\pi\left(\mathscr{T}\left(T, \sigma_{T}\right)\right)$ of the Torelli space $\mathscr{T}\left(T, \sigma_{T}\right)$ by the Torelli modular group $M_{\mathscr{T}}\left(T, \sigma_{T}\right)$.

We have $\mathscr{T}\left(T, \sigma_{T}\right)=\mathscr{J}, M_{\mathscr{T}}\left(T, \sigma_{T}\right)=M_{\mathscr{J}}^{\prime}$, and the exact sequence (7)'. Furthermore, $\pi(\mathscr{J})$ can be shown to be a free group. Fix $\left(\tau_{0}, \tilde{\tau}_{0}\right)$ in $\mathscr{J}$, and let $s$ be a mapping of sets from $M_{\mathscr{J}}^{\prime}$ to $M_{\zeta}\left(T, \sigma_{T}\right)$ such that $j s=1$. Then for each $(\mu, \nu)$ in $M_{\mathscr{J}}^{\prime}, s(\mu, \nu)$ corresponds to a unique homotopy class $\beta$ of curves from $\left(\tau_{0}, \tilde{\tau}_{0}\right)$ to $\left(\mu \tau_{0}, \nu \tilde{\tau}_{0}\right)$, and then each $(\mu, \nu)$ determines an automorphism $\beta(\mu, \nu)$ on $\pi\left(\mathscr{J},\left(\tau_{0}, \tilde{\tau}_{0}\right)\right)$ by $\beta(\mu, \nu): \alpha \rightarrow \beta((\mu, \nu) \alpha) \beta^{-1}$. Note that if $(\mu, \nu)$ keeps $\left(\tau_{0}, \tilde{\tau}_{0}\right)$ fixed, then $1(\mu, \nu): \alpha$ 
$\rightarrow(\mu, \nu) \alpha$ is in Aut $\pi\left(\mathscr{J},\left(\tau_{0}, \tilde{\tau}_{0}\right)\right)$, and $1(\mu, \nu)=\beta(\mu, \nu) \bmod \operatorname{Inn} \pi\left(\mathscr{J},\left(\tau_{0}, \tilde{\tau}_{0}\right)\right)$. Now $\mathscr{J}$ consists of $\boldsymbol{H} \times \boldsymbol{H}$ deleted by a discrete subset of nonintersecting planes, so that $\pi(\mathscr{J})=\pi\left(\mathscr{J} \cup\left\{x_{0}\right\}\right)$, where $\mathscr{J} \cup\left\{x_{0}\right\}$ denotes the one-point compactification of $\mathscr{J}$; i.e., the element $x_{0}$ is identified with the boundary $\partial(H \times H)$ of $H \times H$. Furthermore, $(\mu, \nu): \partial(H \times H) \rightarrow \partial(H \times H)$ for each $(\mu, \nu)$ in $M_{\mathscr{J}}^{\prime}$, so that every element of $M_{\mathscr{J}}^{\prime}$ has $x_{0}$ for a fixed point. If we then take $x_{0}$ (instead of $\left.\left(\tau_{0}, \tilde{\tau}_{0}\right)\right)$ for the base point, the natural homomorphism $M_{\mathscr{J}}^{\prime} \rightarrow$ Out $\pi(\mathscr{J})$ can be lifted to $M_{\mathscr{J}}^{\prime} \rightarrow$ Aut $\pi(\mathscr{J})$ which completes the proof.

\section{REFERENCES}

1. O. Bolza, On binary sextics with linear transformations into themselves, Amer. J. Math. 10 (1888), 47-60.

2. M. Gerstenhaber, On a theorem on Haupt and Wirtinger concerning the periods of a differential of the first kind, and a related topological theorem, Proc. Amer. Math. Soc. 4 (1953), 476-481.

3. J. I. Hutchinson, On the reduction of hyperelliptic functions $(p=2)$ to elliptic functions by a transformation of the second degree, Ph.D. dissertation, University of Chicago, 1895.

4. W. Mangler, Die Klassen von topologischen Abbildungen einer geschlossenen Flache auf sich, Math. Z. 44 (1939), 541-554.

5. H. E. Rauch, A transcendental view of the space of algebraic Riemann surfaces, Bull. Amer. Math. Soc. 71 (1965), 1-39.

6. J. Schiller, Riemann matrices for hyperelliptic surfaces with involutions other than the interchange of sheets, Michigan Math. J. 15 (1968), 283-287.

TEMPLE UNIVERSITY,

Philadelphia, Pennsylvania 